\title{
CARACTERIZACIÓN DE LOS MÉTODOS Y ESTRATEGIAS PE- DAGÓGICAS BÁSICAS EN EL PROCESO DE MOTIVACIÓN DEL NIÑO EN EL NIVEL DE PREESCOLAR EN INSTITUCIO- NES EDUCATIVAS DE LA CIUDAD DE NEIVA
}

\section{CHARACTERIZATION OF BASIC TEACHING METHODS AND STRATEGIES IN THE PROCESS OF MOTIVATION OF PRESCHOOL CHILDREN EDUCATIONAL INSTITUTIONS OF THE CITY OF NEIVA}

\author{
Laura Estefany Celeita Buitrago* María Fernanda Pérez Gutiérrez**
}

\begin{abstract}
Resumen
Este artículo presenta los resultados de la investigación «Caracterización de los métodos y estrategias pedagógicas básicas en el proceso de motivación del niño en el nivel de preescolar», la cual involucró a 10 instituciones educativas 5 del sector oficial y 5 del sector privado. El trabajo de campo implicó la implementación de instrumentos como, la realización de entrevistas a directivos y docentes, registros de observación de las clases y el registro documental.

El objetivo central de la investigación es plantear una propuesta de intervención a las instituciones tanto del sector oficial como del sector privado, que permita consolidar los métodos y las estrategias pedagógicas que garanticen la motivación en el niño de preescolar y permita la obtención de un buen nivel formativo y educativo.
\end{abstract}

Palabras clave: Motivación, método, estrategias, preescolar.

\section{Abstract}

This article presents the results of the research work, "Characterization of the basic teaching methods and strategies in the process of motivation in preschool children", which involved ten schools, five from the public sector and five from the private sector. The field work involved the implementation of tools such as conducting interviews with principals and teachers, classroom observation records and documentary record. The central objective of the research work was to raise an intervention proposal that would allow institutions to consolidate the methods and pedagogical strategies that ensure motivation in preschooler and allow obtaining a good formative and educational level.

Key words: Motivation, methods, strategies, preschool.

Artículo recibido: 04/06/2013 Aprobado: 01/08/2013

* Licenciada en pedagogía infantil. Joven investigadora Colciencias 2011. Investigadora del Grupo Paca categoría A de Colciencias. Email: tichiny2111@hotmail.com

** Licenciada en Pedagogía infantil. Joven investigadora Colciencias 2011. Investigadora del Grupo Paca categoría A de Colciencias. Email: nanita_12_88@hotmail.com

Revista ENTORNOS. Vol. 26, núm. 2. Universidad Surcolombiana. Vicerrectoría de Investigación y Proyección Social, 2013, pp. 231-238 


\section{Introducción}

Una de las funciones principales de la escuela de hoy es cubrir las necesidades básicas de los niños para obtener un mejor aprendizaje, proporcionándoles todo tipo de atención desde la educación, alimentación, transporte, convirtiéndose en un segundo hogar para los niños ya que en ocasiones permanecen más en la escuela que en sus propios hogares. El panorama que deberíamos contemplar, es ver a los niños felices en sus escuelas, saber que están llenos de motivos para levantarse cada mañana e ir a estudiar, que sus experiencias son significativas, que se ponen tristes cuando no hay clases, pero lo que refleja la mayoría de las instituciones educativas es todo lo contrario: las aulas de las instituciones educativas están hacinadas de aburrimiento, los niños no quieren saber de la escuela, se alegran cuando no tienen clases, para muchos es una tortura ir a las instituciones educativas y lo ven como un obligación.

Es necesario conocer el entorno donde se forman los niños, ya que este influye de manera directa e indirecta en el ámbito social, emocional e intelectual, por eso es importante ofrecer un ambiente preparado y adecuado cuidadosamente, creando espacios que fomenten el aprendizaje autónomo, donde se formen seres humanos íntegros, críticos, capaces de pensar libremente y liderar su proceso de formación, aportando creativamente soluciones a problemáticas en aras al desarrollo de la sociedad, aunque respetando el ritmo de desarrollo y aprendizaje de cada ser humano; sin embargo los niños se caracterizan por su curiosidad, su espontaneidad, su sensibilidad y una permanente exploración de su medio; pero la falta de motivación y de interés por aprender es una de las principales causas de los problemas de aprendizaje, un niño motivado aprende con mayor rapidez, eficacia y de manera significativa, los miembros de la comunidad educativa deben tener claro que los contenidos curriculares no son ajenos a esta problemática.

Lo anterior se convirtió en una justificación directa para el desarrollo de la presente investigación que buscó aportar de manera significativa al proceso educativo de los niños de preescolar de las diez instituciones educativas.

\section{Objetivos}

\section{Objetivo general}

Identificar y caracterizar los métodos y estrategias pedagógicas que se implementan en la educación preescolar para incrementar la motivación en el niño, en instituciones educativas oficiales y privadas, que permita la obtención de un buen nivel formativo y educativo.

\section{Objetivos específicos}

- Identificar y caracterizar los diferentes métodos y estrategias pedagógicas empleadas por los docentes para motivar el aprendizaje de los niños en el nivel preescolar en 5 instituciones oficiales y 5 privadas.

- Confrontar las estrategias pedagógicas para incrementar la motivación en el niño, en instituciones educativas oficiales y privadas.

- Plantear una propuesta de intervención al sector oficial como al sector privado que responda a la consolidación de los métodos y estrategias pedagógicas que garanticen la motivación en los niños.

\section{Marco conceptual y contextual}

La educación preescolar es fundamental en el proceso formativo del niño porque durante esta etapa desarrolla habilidades, capacidades y actitudes que deben ser estimuladas para generar aprendizajes significativos. Con la educación preescolar se pretende garantizar las oportunidades básicas para que los niños y niñas se desarrollen en un ambiente sano y seguro. Además debe brindar a las niñas y niños las herramientas necesarias para que tengan la capacidad de interactuar y construir su pensamiento a partir de su contexto.

Es necesario que en el aula de clase, los docentes utilicen métodos y estrategias pedagógicas pertinentes, para motivar al niño en su proceso de aprendizaje. Por lo tanto es importante comprender la conceptualización de los métodos, estrategias, motivación y pedagogía, que permite fortalecer el trabajo investigativo del proyecto Caracterización de los métodos y estrategias pedagógicas básicas en el proceso de motivación del niño en el nivel de preescolar en instituciones educativas de la ciudad de Neiva.

El método dentro del contexto educativo se define, como una secuencia de actividades y acciones que se 
desarrollan para obtener un objetivo ${ }^{1}$. En el proceso aprendizaje los profesores deben conocer como aprenden sus estudiantes, que se les facilita, que les gusta, que les llama la atención y así definir o crear los métodos que se van a desarrollar, el método es uno de los componentes del proceso de enseñanza-aprendizaje, donde los niños adquieren aprendizajes significativos.

En el campo educativo la autora María de los Ángeles Briceño Moreno considera las estrategias como: Aquellas acciones que realiza el maestro con el propósito de facilitar la formación y el aprendizaje de las disciplinas en los estudiantes. Para que no se reduzcan a simples técnicas y recetas deben apoyarse en una rica formación teórica de los maestros, pues en la teoría habita la creatividad requerida para acompañar la complejidad del proceso de enseñanza-aprendizaje ${ }^{2}$. Por su parte, María Parra Pineda, en el documento Manual de estrategias de enseñanza aprendizaje precisa que las estrategias son: procedimientos utilizados por el docente para promover aprendizaje significativo que implican actividades conscientes y orientadas a un fin ${ }^{3}$. De acuerdo a los conceptos planteados de las autoras, se presenta una relación coherente con las estrategias pedagógicas, se definen como un conjunto de acciones o actividades que están enmarcadas en la construcción del aprendizaje del niño, es el elemento fundamental en el proceso de enseñanza, ya que permite mantener la atención en ellos y el interés de las actividades a realizar.

El concepto de motivación, según Alejandra Zepeta Rojas en su artículo Motivación, liderazgo y comportamiento organizacional, conceptúa como: el impulso que conduce a una persona a elegir y realizar una acción entre aquellas alternativas que se presentan en una determinada situación. En efecto, la motivación está relacionada con el impulso, porque este provee eficacia al esfuerzo colectivo orientado a conseguir los objetivos de la empresa, estimula al individuo a la búsqueda continua de mejores situaciones a fin de realizarse profesional y personalmente, integrándolo en la comunidad donde su acción cobra significado 4 .
Por consiguiente existe una relación directa entre la conducta y los factores externos de la persona se busca satisfacer las necesidades básicas y a la vez alcanzar los objetivos propuestos, este proceso se puede evidenciar en la ejecución de una actividad, cuando una persona siente interés o pierde el interés hacia la misma, las emociones juegan un papel importante en la motivación porque depende de la persona y los estímulos del entorno.

El doctor Antoni Ballester Vallori (2002), en su libro «El aprendizaje significativo en la práctica», menciona la importancia de la motivación en el aprendizaje de los estudiantes referencia algunos fundamentos pedagógicos que sustenta los psicólogos Ausubel, Novak y Hanesian frente el aprendizaje significativo que relaciona la motivación y factores externos en el enriquecimiento y éxito de la tarea, orientada bajo la asesoría del docente, es conveniente señalar que las motivaciones internas no se utilizan en el aula de clase ya que los docentes utilizan las recompensas para incrementar la motivación en el aula, sin embargo aclara que se deben implementar la motivación intrínseca para despertar el interés propio por la tarea.

El ser humano es un todo y requiere una visión global, el conocimiento que se imparte está fragmentado, por materias, logros, estándares, competencias, horas, porque lo que importa realmente son las calificaciones, las notas, al profesor no le interesa en lo más mínimo si el estudiante aprende, lo importante es promoverlo al grado siguiente, las personas aprenden lo que le gusta, lo que no le gusta lo aprenden de memoria, solo para presentar una evaluación, sacar una nota o recibir felicitaciones pero a los pocos días la información ha sido olvidada.

Pablo Lipnizky fundador del Colegio Montessori del Municipio la Calera expresa «Preparamos al hombre y a la mujer para la vida por medio de la auto educación, cultivando en el individuo el deseo natural de aprender permitiendo su crecimiento como seres íntegros, libres y felices, agregando valor a la humanidad». En

1. Altamirano. C. Cuauhtémoc, Medina. R. María del Carmen y García M. José Francisco. Definición de Método. CECTE. Centro de Estudios en Comunicación y Tecnologías Educativas.

2. Briceño-Moreno, María de los Ángeles, (2008). Propuesta de estrategias pedagógicas. Educación y educadores. Universidad de la Sabana. Vol. 11.

3. Parra Pineda María Doris, (2003). Manual de estrategias de enseñanza/aprendizaje, (en línea). SENA Regional Antioquia. Disponible en http:/l www.cepefsena.org/documentos/METODOLOGIAS\%20ACTIVAS.pdf

4. Zepeta Rojas Alejandra. (2008, marzo). Motivación, liderazgo y comportamiento organizacional. Universidad del Valle de México, (en línea). Disponible en: http://www.losrecursoshumanos.com/phpscript/descargar_pdf.php?id=1941 
sus planteamientos pedagógicos María Montessori establece que el ambiente y el amor son fundamentales para la formación trabajando la libertad con responsabilidad, además resalta que la mente de los niños tiene una capacidad maravillosa e infinita, porque absorben como «esponjas» toda la información que requieren y necesitan para su diario vivir.

Lipnizky también explica que un punto fundamental en la filosofía Montessori es educar niños responsables de sus acciones y no víctimas del entorno, niños que se expresen con libertad, dignidad, independencia y responsabilidad, permitiendo que se desarrolle en áreas de conocimiento a nivel; cognitivo, social, cultural, afectivo y emocional donde comprendan la realidad y el entorno que los rodea, creando ambientes socializadores $y$ de interés.

La escuela como proceso de transformación debe propiciar espacios de aprendizaje que busquen la construcción del conocimiento a partir de la interacción y participación, procesos que se trabajan con la voz de los actores sociales (profesores, padres de familia y niños) como alternativa para lograr cambios en las prácticas pedagógicas.

Hoy en día la escuela se encuentra bajo un modelo pedagógico que aporta a la formación integral del niño y a un aprendizaje significativo; no obstante en las prácticas pedagógicas se desarrolla una metodología tradicional y sistemática que afecta el aprendizaje y el rendimiento académico del niño. Las estrategias y métodos no adecuados implementados por los profesores hacen que el niño se desmotive en las actividades propuestas.

El modelo de Reggio Emilia se encuentra inspirado en la pedagogía de Loris Malaguzzi, que sustenta una metodología dinámica y transformadora. Uno de los objetivos que persigue el modelo es diseñar una escuela activa que se convierta en un lugar de investigación y experimentación teniendo en cuenta intereses y necesidades del niño; en el desarrollo de esta metodología participan profesores, padres de familia y agentes del entorno, comprometidos con la ejecución de los proyectos.
El papel que asume el profesor es el de proporcionar las herramientas necesarias para fortalecer el proyecto. Los niños son los protagonistas del proceso, ya que ellos son quienes proponen las actividades a desarrollar, participan, reflexionan y buscan dar solución a los problemas.

Esta metodología busca crear ambientes agradables que generen cambios y desarrollos significativos en el aprendizaje de los niños, destacando la importancia de los diferentes agentes y las relaciones personales que se dan en el aula. Igualmente el proyecto propicia la participación de los padres de familia con el fin de que conozcan el desempeño de los niños y las actividades que desarrollan en la escuela.

Es importante mencionar que la educación preescolar de hoy, debe asumir una propuesta alternativa que beneficie el desarrollo individual del niño y promueva las relaciones sociales. Por lo anterior los profesores deben propiciar espacios de participación, socialización, reflexión y concertación entre los niños que les permita construir identidad-cultural relacionada con el contexto al que pertenezcan.

De igual manera es necesario que durante este proceso se desarrollen elementos fundamentales en el aula de clase, según Marco Raúl Mejía señala dos aspectos: «El diálogo de saberes se construye como política, funciona a través de los lenguajes, los conocimientos, las interacciones, las formas disciplinarias, las dinámicas y dispositivos. Para construir una acción fruto del intercambio y la visibilización de la posibilidad de un futuro distinto». Y la negociación cultural "ocurre en un lugar intermedio entre el aprendizaje clásico y un tipo de aprendizaje reconstructivo que reconstruye vivencias a partir de la realidad, genera acciones que propician nuevos intereses, construye una motivación con capacidad de acción y permite necesidades de formación todo ello para hacer posible la transformación» ${ }^{5}$.

La escuela como organismo educativo debe desarrollar acciones pedagógicas donde el profesor propicie espacios de interacción con los niños, para debatir, pensar y confrontar las problemáticas que pasan en el contexto. Este trabajo se realiza con el compromiso de la comunidad educativa, profesores y padres de familia, con el fin de garantizarles a los niños un mejor futuro.

5. Mejía J. Marco Raúl, (2012). Educaciones y pedagogías críticas desde el Sur. Editorial Planeta Paz. Bolivia. 


\section{Metodología}

La naturaleza de la investigación fue etnográfica, su proceso se centró en lo cualitativo, el principal elemento fue la observación con una descripción amplia y enriquecedora de los contextos y actividades educativas realizadas en las instituciones educativas, con lo anterior se registra aspectos importantes que ayudan a confrontar las diversas concepciones de los autores, que permite hacer un análisis descriptivo de las estrategias y métodos pedagógicos que utilizan los docentes en el aula escolar. Este tipo de técnica vincula el mayor número de agentes de la comunidad educativa, los directivos por tener conocimiento en los procesos que se desarrollan en la institución, los docentes se consideran acompañantes y guías permanentes del proceso educativo y los niños porque son los principales actores en el proceso de enseñanza-aprendizaje.

\section{Población y muestra}

Para el desarrollo de la investigación se eligieron diez instituciones de la ciudad de Neiva, teniendo en cuenta los siguientes requisitos:

- Cinco instituciones del sector oficial.

- Cinco instituciones del sector privado.

- Aval de las instituciones para realizar la investigación.

- El grado de transición.

Las audiencias que sirvieron de fuente de información estuvieron constituidas por 4 rectoras, 6 coordinadores y 16 docentes.

\section{Instrumentos}

Para realizar el trabajo de campo fue necesario elaborar los instrumentos que permitieron recoger información de las Instituciones objeto de estudio. Se elaboraron los instrumentos pertinentes como Registro de Observación, Registro Documental, Entrevistas a directivos y a docentes relacionada a la metodología y a los objetivos del proyecto.

\section{Análisis documental}

En el desarrollo de la investigación se realizó un análisis detallado sobre la educación preescolar en el contexto internacional, nacional y local, además la conceptualización de métodos, estrategias pedagógicas y motivación en el aula, que sustentará el planteamiento del problema. Luego se procedió a una revisión documental del PEI en las Instituciones del sector oficial y el sector privado. Posteriormente se efectuó un análisis detallado de las respuestas dadas por parte de los docentes y directivos frente a las preguntas realizadas, el procesamiento de la información obtenida de los instrumentos aplicados en las Instituciones objeto de estudio, permitieron la elaboración de una propuesta de intervención que respondiera a la consolidación de los métodos y estrategias pedagógicas que garanticen la motivación en los niños.

\section{Resultados y consideraciones finales}

Una vez culminado el proyecto de investigación «Caracterización de los métodos y estrategias pedagógicas básicas en el proceso de motivación del niño en el nivel de preescolar en instituciones educativas de Neiva» y después de analizada y procesada la información obtenida con la aplicación de los instrumentos (Entrevista a Directivos, Entrevista a Docentes, Registro de Observación y Registro Documental) se plantearon las siguientes conclusiones:

1. Los recursos didácticos y pedagógicos con los que cuentan las instituciones privadas no son utilizados por parte de las docentes en el desarrollo de las actividades educativas ya que se limitan a utilizar el tablero, la cartilla y el cuaderno. Mientras que las instituciones oficiales cuentan con escasos recursos pedagógicos, siendo este un elemento fundamental para la motivación del niño en su proceso de aprendizaje.

2. Los espacios como bibliotecas, salas múltiples, parques y espacios abiertos brindados por las instituciones educativas oficiales y privadas no son aprovechados adecuadamente. Las docentes optan más bien por desarrollar su trabajo educativo dentro del salón de clases convirtiéndose en una práctica reiterativa.

3. Se evidenció que la escasa disponibilidad de espacio en las aulas de clase de la mayoría de las instituciones privadas impide el normal desarrollo de las actividades educativas. 
4. Todo el proceso educativo realizado por las docentes de las instituciones tanto del sector público como del sector privado, está regido bajo los parámetros directos de las docentes, siendo ellas las protagonistas del proceso de enseñanza-aprendizaje, restringiendo la participación de los niños a simples receptores pasivos de información y limitándolos a hacer lo que la maestra considera pertinente. Como resultado forman a un niño sin interés, sin el deseo de aprender, apagándole la llama de la creatividad, siendo esta una de sus características innatas, al no tener en cuenta las diferencias individuales ni los diversos estilos de aprendizaje.

5. En cuanto a las estrategias de motivación implementadas por las docentes en el sector privado (rondas y ejercicios de atención), y en el sector oficial (juegos y canciones) no son suficientes para que los niños tengan un verdadero interés por aprender debido a su poca motivación y distracción durante el desarrollo de la clase.

6. Las docentes tanto del sector oficial como del privado expresaron impulsar proyectos de aula, teniendo en cuenta los intereses y las necesidades de los niños. No obstante se evidenció que en la mayoría de las instituciones de los dos sectores no desarrollan este tipo de proyectos, por cuanto los contenidos ya están programados y planeados por parte del docente.

Cabe resaltar que en el registro de observación se evidenció que en dos instituciones educativas del sector oficial desarrollan proyectos de aula teniendo en cuenta las pautas respectivas, donde se lleva a cabo un trabajo lúdico y dinámico con los niños.

A pesar de que las docentes tienen claro la importancia de los proyectos de aula en el desarrollo de las actividades estos no se llevan a la práctica. Las docentes del sector oficial manifestaron que los proyectos de aula promueven aprendizajes significativos en los niños argumentando que «Los niños aprenden de sí mismos y de sus experiencias», mientras que el sector privado expresó «La participación activa por parte de los niños».

Pese a que dentro de los PEl los enfoques están muy marcados a la formación integral y la cons- trucción de aprendizajes significativos, estos se quedan solamente en el papel ya que en la medida que avanza el calendario académico vemos que los intereses y las necesidades de los niños son relegados por actividades como las planas y las tareas.

7. En relación a las cualidades del docente se observó que la mayoría tienen una buena relación con los niños, basada en afecto y confianza, sin embargo en el sector oficial se evidenció que algunas docentes mantienen una relación autoritaria. Asimismo se registró que a pesar de que existen buenas relaciones con los niños, las docentes no promueven espacios de socialización, donde los niños participen, reflexionen, planteen preguntas y sean los protagonistas de la clase. Siendo este un factor fundamental para incrementar la motivación en su proceso formativo.

8. Los incentivos que utilizan los docentes del sector oficial para motivar a los niños durante el desarrollo de las actividades son las caritas felices y los dulces, por el contrario el sector privado implementa los elogios resaltando las cualidades de los niños de preescolar. Es importante destacar que los incentivos verbales tienen un efecto positivo en el comportamiento y el aprendizaje de los niños porque permiten a que se interesen por aprender.

9. La mayoría de los docentes de las instituciones que hacen parte del trabajo investigativo afirmaron tener en cuenta los diferentes ritmos de aprendizaje de sus estudiantes en el proceso educativo y dedican unos días especiales en la semana para atender las dificultades que presentan algunos de los niños. Sin embargo el equipo investigador durante el trabajo de campo no evidenció el desarrollo de estos procesos.

10. Aunque las docentes del sector oficial y el sector privado manifestaron conocer las condiciones socioeconómicas y culturales de sus estudiantes porque durante el proceso de matrícula los padres de familia diligencian un formato donde especifican el estrato social y el estado civil, se considera que la información obtenida por este medio resulta insuficiente; no hay un acompañamiento real por parte de la docente donde pueda constatar la información obtenida y el contexto familiar del niño, 
dada la alta incidencia que estas condiciones tienen en los ambientes de aprendizaje y en los desarrollos biológicos, sicológicos y sociales de los niños.

\section{Propuesta de intervención alternativa}

Métodos y estrategias pedagógicas para incrementar la motivación en los niños del nivel preescolar

De acuerdo a los resultados encontrados a través del análisis de los instrumentos aplicados en las diez (10) instituciones educativas, cinco oficiales y cinco privadas de la ciudad de Neiva, se evidenció que la mayoría de profesores no utilizan los métodos y estrategias pedagógicas pertinentes para motivar al niño en su proceso de aprendizaje. Por lo tanto se ve la necesidad de realizar una propuesta de intervención que contribuya a la formación integral del niño y supere las falencias encontradas.

Es preciso señalar que la propuesta de intervención que se plantea en el proyecto de investigación tiene aspectos fundamentales de la pedagogía critica, la educación popular, la filosofía de Paulo Freire y el modelo pedagógico de Reggio Emilia que busca consolidar los métodos y estrategias pedagógicas para incrementar la motivación de los niños del nivel de preescolar.

Es necesario mencionar los elementos fundamentales que deben tener en cuenta las instituciones educativas que hicieron parte del trabajo investigativo:

En la escuela actual se siguen desarrollando los mismos métodos tradicionales, transmisión de conocimientos y memorización de conceptos considerados como educación bancaria. Según Paulo Freire: «el educador el cual conduce al educando en la memorización mecánica de los contenidos.

Los educandos son así una suerte de «recipientes» en los que se «deposita» el saber. El educador no se comunica sino que realiza depósitos que los discípulos aceptan dócilmente. El único margen de acción posible para los estudiantes es el de archivar los conocimientos» ${ }^{6}$. Donde se convierten en actores pasivos del proceso educativo que afectan su desempeño académico. Es importante que la escuela de hoy sea intervenida en todos sus aspectos académicos, curriculares e institucionales para contribuir a un mejor nivel formativo del niño.

Las instituciones educativas deben promover en los niños y niñas costumbres, valores y principios de su cultu$\mathrm{ra}$, donde se integren a la realidad social, aprendiendo de una manera dinámica y reflexiva sobre las situaciones que se presentan en su entorno.

Desde una perspectiva crítica es necesario que la educación preescolar se convierta en un proceso participativo y transformador que generen cambios en los métodos y estrategias pedagógicas que implementan los profesores para garantizar la motivación de los niños donde se desarrollen espacios de socialización e interacción que permite la construcción del conocimiento a partir de las experiencias.

Esto permite que la educación preescolar aporte a los niños y niñas aspectos sustantivos en el proceso educativo, donde se respeten los intereses y necesidades de los niños que fortalecen su desarrollo integral.

Los profesores de las instituciones educativas del sector oficial y el sector privado deben conocer los contextos y condiciones socioeconómicas y culturales de sus estudiantes donde se realice un trabajo de acompañamiento con padres de familia y niñas que permite crear acciones pedagógicas para su proceso de aprendizaje.

Es importante que el profesor propicie espacios de participación, reflexión y diálogo para que los niños planteen preguntas y construyan sus propias respuestas, el profesor no es el que aporta las respuestas, sino que ayuda a orientarlos, brindándoles elementos primordiales en el proceso de aprendizaje. Los intereses y necesidades de los niños deben tenerse en cuenta en el desarrollo de las actividades y situaciones que ellos planteen porque parten de la realidad y de la experiencia del entorno.

La pedagogía crítica señala la construcción de procesos de innovación y transformación pedagógica, ligados a las realidades contextuales. (Mejía J. Marco 2012) donde los profesores desde la socialización, analicen, comprendan y transformen los planteamientos de los ni-

6. Freire Paulo. (1970). Pedagogía del Oprimido. Editores S.A de C.V. México. 
ños, en busca de soluciones y alternativas para contribuir en su proceso educativo y formación integral de ellos.

Es necesario que las instituciones educativas oficiales y privadas desarrollen proyectos a través de la metodología de Reggio Emilia que busca crear ambientes agradables donde el niño interactúe, comprenda, experimente, y explore la realidad, para resolver sus inquietudes. La participación de la familia, docentes y comunidad educativa es primordial en el proceso del aprendizaje del niño, ya que participan en el desarrollo de los proyectos y a su vez aprenden con ellos.

Los proyectos, se fundamentan en experiencias a partir de los intereses y necesidades de los niños, estos intereses deben conocerlos los profesores para integrarlos a los objetivos, un aspecto primordial en la ejecución de los proyectos, es la motivación de los niños por aprender.

Es importante resaltar que las escuelas de Reggio Emilia no tiene currículo ni programaciones, pero los buscan transformándolos en otra cosa, viviendo con los niños, trabajando con la certeza, la incertidumbre y con lo nuevo. La ignorancia es la que los impulsa a investigar partiendo de las ideas, sugerencias, interrogantes y problemas que surgen de los niños ${ }^{7}$. Permitiendo que los intereses y la curiosidad de los niños sean el norte del proceso formativo y el quehacer del día a día en la escuela.

Un factor fundamental en el proceso educativo es la relación profesor-estudiante, pues el profesor es quien debe formar al niño en su integridad como persona, fundando en ellos valores y enseñanzas que los guiarán toda su vida, respetando las particularidades de cada uno de ellos, para no homogenizar y encajar a todos en un solo molde. El objetivo es que el niño sienta y sepa que el profesor va a su lado, que lo acompaña y que lo guía.

Para finalizar se considera pertinente el desarrollo de capacitaciones y socializaciones de experiencias significativas en la educación para la primera infancia, ya que esto se convierte en una herramienta fundamental para el desarrollo de los procesos en las actividades educativas. Estos encuentros le permiten al maestro tener una visión más amplia y enriquecedora sobre los procesos de enseñanza y aprendizaje.

\section{Referencias bibliográficas}

Altamirano, C. Cuauhtémoc, Medina. R. María del Carmen y García M. José Francisco. Definición de Método. CECTE. Centro de Estudios en Comunicación y Tecnologías Educativas.

Briceño Moreno, María de los Ángeles. Propuesta de estrategias pedagógicas. Educación y educadores. Universidad de la Sabana. Vol. 11, 2008.

Parra Pineda María Doris. Manual de estrategias de enseñanza/aprendizaje, (en línea). SENA Regional Antioquia. Disponible en: http://www.cepefsena.org/documentos/METODOLOGIAS\%20ACTIVAS.pdf, 2003.

Zepeta Rojas Alejandra. Motivación, liderazgo y comportamiento organizacional. Universidad del Valle de México, (en línea). Disponible en: http://www.losrecursos humanos.com/phpscript/descargar_pdf.php? id=1941, Marzo, 2008.

7. Educación Alternativa.org. Metodología Reggio Emilia. Disponible en: http://educacionalternativa.edublogs.org/articulos/metodologia-reggio-emilia/ 